This is the accepted version of the following article: Green, H. J., Tefay, M., \& Mihuta, M. E. (2018). Feasibility of small group cognitive rehabilitation in a clinical cancer setting. Psycho-Oncology, 27, 1341-1343. doi:10.1002/pon.4600, which has been published in final form at https://doi.org/10.1002/pon.4600. This article may be used for non-commercial purposes in accordance with the Wiley Self-Archiving Policy [ https://authorservices.wiley.com/author-resources/Journal-Authors/licensingopen-access/open-access/self-archiving.html ].

\title{
Feasibility of Small Group Cognitive Rehabilitation in a Clinical Cancer Setting
}

\author{
Heather J. Green ${ }^{1}$, Merilyn Tefay ${ }^{2}$, Mary E. Mihuta ${ }^{1}$
}

${ }^{1}$ Menzies Health Institute Queensland and School of Applied Psychology, Griffith University, Southport, QLD, Australia

${ }^{2}$ Mater Health Services, Brisbane, QLD, Australia

Correspondence:

Dr Heather Green, School of Applied Psychology, Griffith University, Southport, QLD, Australia

Email: h.green@griffith.edu.au

Funding Information: Funders were Mater Foundation; School of Applied Psychology, Griffith University; and Department of Science, Information Technology and Innovation, Queensland Government

Conflicts: None

Keywords: Cancer, oncology, cognitive rehabilitation, allied health, group 


\section{Key Points}

- Cancer related cognitive impairment is often reported by patients treated with chemotherapy for breast cancer.

- A previously successful cognitive rehabilitation intervention for this issue was trialled in a clinical setting.

- Approximately $20 \%$ of patients attending the centre per year expressed interest with approximately $10 \%$ attending the program.

- Participants were highly satisfied and improved significantly on executive functioning performance.

- This small group cognitive rehabilitation approach appears feasible for clinical centres. 


\section{Background}

Cancer-related cognitive impairment (CRCI) has been demonstrated following adjuvant chemotherapy for breast cancer. ${ }^{1}$ Cross sectional studies have reported frequencies of CRCI up to $75 \%$ and impacting areas of cognition such as memory, attention, executive functioning, and processing speed. ${ }^{2}$ Cognitive changes may endure for up to 10 years following treatment and may impact quality of life (QoL), relationships, and occupational functioning. ${ }^{3}$

Although impact of CRCI is increasingly recognised, relatively few published studies have investigated CRCI interventions ${ }^{4}$. "Responding to Cognitive Concerns" $(\mathrm{ReCog})$ is a cognitive rehabilitation (CR) intervention developed to address CRCI in adult cancer survivors using skills training and compensatory strategies and has been evaluated in two previous studies. In the first study, intervention participants improved significantly more than waitlist participants in overall neuropsychological performance, immediate memory, delayed memory, and visuospatial/constructional performance. ${ }^{5}$ Intervention participants also reported improved perceptions of cognitive and social functioning. A later randomised controlled trial demonstrated that intervention participants improved significantly more than waitlist participants in processing speed, perceived cognitive impairment, and cognitive self-efficacy. ${ }^{6}$ Previous studies showed moderate to large within-group effect sizes. ${ }^{5,6}$

However, both these studies were conducted in research settings. There is considerable interest in implementing psycho-oncology CR interventions clinically, but such a trial has not yet been reported. This study aimed to evaluate the feasibility and effect size of implementing ReCog within a clinical setting. This is an important step in assessing the suitability of implementing such interventions clinically, since there are currently no specific evidence-based interventions that have been recommended for managing $\mathrm{CRCI}$ in routine practice.

\section{Method}

\subsection{Participants}

Twenty-seven female participants enrolled following university and hospital ethics approval (HREC/15/MHS/10) at Mater Cancer Care Centre, Brisbane, Australia, during June 2015 to November 2016. A priori power calculations estimated 27 participants for $80 \%$ power to detect within-participant effect size $d=0.5$ as per 
previous ReCog research ${ }^{5,6}$ (one-tailed testing, $\alpha=0.05$ ). Eligibility criteria were (a) breast cancer history, (b) treatment with chemotherapy, (c) completed primary treatment 6 to 60 months prior, (d) no anticipated cancer treatments apart from hormonal medications, (e) self-reported cognitive complaints, (f) able to speak, read, and write English fluently.

\subsection{Procedure}

Women treated with chemotherapy for breast cancer at the centre in the previous 2 years were mailed and could elect further information or no further contact. Treating health professionals also discussed the study with patients attending the centre. Potential participants contacted researchers and underwent telephone screening to assess eligibility and provide demographic/clinical data. Enrolled participants completed baseline assessment, participated in the 4-week intervention in groups of 3 to 9 participants, and then completed post-intervention assessment.

\subsection{Intervention}

ReCog is a manualised intervention using self-regulatory CR and cognitive behavioural principles. ReCog involves skills training, compensatory strategies, group discussion, and between-session homework. Session topics are: (1) aging, health, cancer and cognitive function; (2) memory; (3) attention; and (4) fatigue, emotions and cognition. Facilitators completed a two-hour training workshop with the manual's co-author. Weekly two-hour group sessions were co-facilitated by an occupational therapist and a second occupational therapist or occupational therapy student.

\subsection{Measures}

\subsubsection{Participant satisfaction}

Primary outcomes were recruitment rate and participant satisfaction measured with ratings of (a) satisfaction with the program, (b) change in cognitive functioning, and (c) likelihood of recommending the program to friends with similar problems on a 5-point Likert scale, plus overall program rating from 1(very poor) to 10(excellent). Participants also commented on most enjoyable, least enjoyable, and otherwise noteworthy aspects of the program. This measure was used in previous $\mathrm{ReCog}$ studies $^{5,6}$.

\subsubsection{Other measures}

Self-reported cognition was assessed with the 37-item Functional Assessment of Cancer Therapy-Cognitive Scale (FACT-Cog3). ${ }^{7}$ The measure contains four subscales: perceived cognitive impairments (PCI), perceived cognitive abilities 
(PCA), comments from others (OTH), and impact on QoL (IQL). The online WebNeuro battery comprised 11 computerised tasks assessing seven objective cognitive domains. ${ }^{8}$ QoL was evaluated with the 30-item European Organisation for Research and Treatment of Cancer QoL Questionnaire Core 30 version 3 (EORTCQLQ-C30) ${ }^{9}$ and distress with the 10-item Kessler Psychological Distress Scale $(\mathrm{K} 10){ }^{10}$

\section{Results}

Fifty-six volunteers were screened: 31 respondents from a mailout to 225 patients and 25 invited by treating staff. Of these 56, 27 enrolled, 25 were unable (timing/work/transport/other issues), and 4 were ineligible. Two of 27 participants withdrew before baseline assessment (T1) for scheduling conflicts, yielding 25 participants who completed T1 assessments and the intervention. Six participants did not respond to post-treatment (T2) assessment reminders and their T1 scores were carried forward.

Participants' mean age was 49.9 years $(\mathrm{SD}=10.4)$; they completed 14.8 years of education ( $\mathrm{SD}=2.9$ ); and finished primary treatment 14.3 months previously $(\mathrm{SD}=9.2$ ). All participants had surgery for treatment, $96 \%$ had chemotherapy, $81 \%$ had radiotherapy, and 58\% received hormone therapy.

As Table 1 shows, all participants endorsed at least moderate satisfaction with ReCog and believed their cognitive problems had improved, and 95\% would recommend the program. Average program rating was $8.68 / 10(\mathrm{SD}=1.20)$. Feedback on most valued components most often endorsed program content $(81 \%$ of participants) and group interaction (50\% of participants).

Baseline FACT-Cog3 mean scores (Table 2) were impaired relative to a previous demographically-similar community sample. ${ }^{6}$ WebNeuro mean Z scores showed significant baseline impairment relative to the WebNeuro standardisation sample $^{8}$ on Working Memory and Impulsivity. From baseline to post-intervention, participants demonstrated significant one-tailed within-participant t-test improvement in some of the domains (Table 2). Two of four FACT-Cog3 subscales improved: PCI, $t(23)=-1.72, p=.050$, and IQL, $t(23)=-2.93, p=.004$. Improved physical functioning, $t(23)=-1.86, p=.038$, role functioning, $t(23)=-2.07, p=.025$, cognitive functioning, $t(23)=-1.90, p=.036$, and social functioning $t(23)=-2.02, p=.028$ were reported on EORTC-QLQ-C30. Psychological distress reduced, $t(23)=2.58, p=.009$. On objective 
cognitive functioning, participants demonstrated improved information processing efficiency, $t(24)=-2.60, p=.008$, and executive functioning, $t(24)=-3.28, p=.002$. Significant effects showed small to moderate effect sizes (Table 2). Using Bonferroni adjusted significance testing for 21 comparisons ( $p=.002)$, only the executive functioning improvement remained significant.

\section{Conclusions}

Results showed it was feasible to deliver group CR within clinical practice. Participants were highly satisfied, comparable to previous ReCog research ${ }^{5}$. Group interaction was valued by many participants, differing from online cognitive training which is also evidence-based but lacks this social component ${ }^{4}$. After correction for multiple comparisons, participants improved significantly on a measure of objective executive functioning. Effect sizes suggested modest improvements also in selfreported cognition, QoL, and distress, but larger samples are needed to test replicability. Limitations include use of an English speaking, highly educated sample from a single centre.

These findings imply that small group CR interventions are effectively translatable into clinical practice. Consideration could be given to providing $\mathrm{CR}$ intervention earlier than 6 months post treatments, since returning to work impeded participation for many volunteers. 


\section{REFERENCES}

1. Ono M, Ogilvie JM, Wilson JS, et al. A meta-analysis of cognitive impairment and decline associated with adjuvant chemotherapy in women with breast cancer. Front Oncol. 2015;5:59.

2. Ahles TA, Root JC, Ryan EL. Cancer- and cancer treatment-associated cognitive change: an update on the state of the science. J Clin Oncol. 2012;30(30):3675-3686.

3. Boykoff N, Moieni M, Subramanian SK. Confronting chemobrain: an in-depth look at survivors' reports of impact on work, social networks, and health care response. J Cancer Surviv. 2009;3(4):223-232.

4. Bray VJ, Dhillon HM, Bell ML, et al. Evaluation of a web-based cognitive rehabilitation program in cancer survivors reporting cognitive symptoms after chemotherapy. J Clin Oncol. 2017;35(2):217-225.

5. Schuurs A, Green HJ. A feasibility study of group cognitive rehabilitation for cancer survivors: enhancing cognitive function and quality of life.

Psychooncology. 2013;22:1043-1049.

6. King S, Green HJ. Psychological intervention for improving cognitive function in cancer survivors: a literature review and randomized controlled trial. Front Oncol. 2015;5:1-18.

7. Wagner LI, Sweet J, Butt Z, Lai J, Cella D. Measuring patient self-reported cognitive function: Development of the Functional Assessment of Cancer Therapy - Cognitive Function Instrument. J Support Oncol. 2009;7:W32-W39.

8. Silverstein SM, Berten S, Olson P, et al. Development and validation of a World-Wide-Web-based neurocognitive assessment battery: WebNeuro. Behav Res Methods. 2007;39:940-949.

9. Aaronson NK, Ahmedzai S, Bergman B, et al. The European Organization for Research and Treatment of Cancer QLQ-C30: a quality-of-life instrument for use in international clinical trials in oncology. J Natl Cancer Inst. 1993;85:365-376.

10. Kessler RC, Andrews G, Colpe LJ, et al. Short screening scales to monitor population prevalences and trends in non-specific psychological distress. Psychol Med. 2002;32:959-976. 
TABLE 1 Participant satisfaction

\begin{tabular}{lcc}
\hline Item (range 1-5) & M (SD) & $\begin{array}{c}\text { Endorsed moderately } \\
\text { or greater, \% }\end{array}$ \\
\hline $\begin{array}{l}\text { How satisfied are you with the treatment } \\
\text { you received through the group program? }\end{array}$ & $4.53(0.51)$ & 100 \\
$\begin{array}{l}\text { To what extent have your cognitive } \\
\text { problems changed since the group } \\
\text { treatment? }\end{array}$ & $4.21(0.42)$ & 100 \\
$\begin{array}{l}\text { If you had a friend with a similar problem, } \\
\text { how likely is it that you would recommend } \\
\text { they attend this program? }\end{array}$ & $4.47(0.61)$ \\
& \\
Item (range 1-10)
\end{tabular}

Overall, how would you rate the program?

$8.68(1.20)$

100 
TABLE 2 Outcomes on secondary measures

\begin{tabular}{|c|c|c|c|c|}
\hline Measure $^{a}$ & $\mathbf{T 1}$ & $\mathbf{T} 2$ & 1-tailed $p$ & $d$ \\
\hline \multicolumn{5}{|l|}{ FACT-Cog3 } \\
\hline PCI (0-72) & $37.08(15.72)$ & $42.29(14.19)$ & .050 & .33 \\
\hline PCA $(0-28)$ & $18.75(6.86)$ & $17.79(6.57)$ & .845 & -.14 \\
\hline OTH (0-16) & $12.46(3.55)$ & $12.83(4.05)$ & .186 & .11 \\
\hline IQL (0-16) & $8.92(4.92)$ & $10.67(4.43)$ & .004 & .36 \\
\hline \multicolumn{5}{|l|}{ WebNeuro } \\
\hline Verbal memory & $-0.05(0.90)$ & $-0.07(0.96)$ & .569 & -.02 \\
\hline Working memory & $-0.59(1.41)$ & $-0.28(1.03)$ & .054 & .22 \\
\hline Attention & $-0.23(0.81)$ & $-0.20(0.85)$ & .402 & .04 \\
\hline Information processing & $-0.36(0.62)$ & $-0.15(0.59)$ & .008 & .35 \\
\hline Executive function & $0.03(0.61)$ & $0.56(0.69)$ & .002 & .87 \\
\hline Response speed & $-0.14(0.84)$ & $-0.06(1.04)$ & .357 & .09 \\
\hline Impulsivity & $-0.46(0.43)$ & $-0.32(0.55)$ & .090 & .33 \\
\hline \multicolumn{5}{|l|}{ EORTC-QLQ-C30 (0-100) } \\
\hline Physical function & $84.44(15.19)$ & $87.22(16.67)$ & .038 & .18 \\
\hline Role function & $77.78(21.80)$ & $82.64(21.69)$ & .025 & .22 \\
\hline Emotional function & $64.58(24.85)$ & $68.06(24.66)$ & .164 & .14 \\
\hline Cognitive function & $55.56(24.41)$ & $61.81(28.44)$ & .036 & .26 \\
\hline Social function & $60.42(30.62)$ & $70.14(32.59)$ & .028 & .32 \\
\hline Global QoL & $62.85(17.72)$ & $66.67(18.71)$ & .063 & .22 \\
\hline Fatigue $^{\mathrm{b}}$ & $43.52(21.21)$ & $37.50(21.19)$ & .025 & .28 \\
\hline Nausea/vomiting ${ }^{\mathrm{b}}$ & $3.47(6.91)$ & $6.94(10.90)$ & .989 & -.50 \\
\hline Pain ${ }^{\mathrm{b}}$ & $33.33(25.54)$ & $25.69(24.56)$ & .031 & .30 \\
\hline Distress $^{\mathrm{b}}(10-50)$ & $22.79(8.63)$ & $20.00(7.50)$ & .009 & .32 \\
\hline
\end{tabular}

${ }^{\mathrm{a}} \mathrm{Mean}(\mathrm{SD})$ and possible ranges are shown for self-report measures, and Z-scores for WebNeuro domains. Positive effect sizes $(d)$ represent improved functioning.

${ }^{\mathrm{b}}$ Lower scores indicate better functioning 